\title{
Solar Magnetic Polarity Effect on Neutron Monitor Count Rates from Latitude Surveys Versus Antarctic Stations
}

\author{
K. Poopakun, ${ }^{a, *}$ W. Nuntiyakul, ${ }^{a}$ D. Ruffolo, ${ }^{b}$ P. Evenson, ${ }^{c}$ J. Peng, ${ }^{d}$ \\ P. Chuanraksasat, ${ }^{e}$ M. L. Duldig, ${ }^{f}$ J. E. Humble ${ }^{f}$ and S. Oh ${ }^{g}$ \\ ${ }^{a}$ Department of Physics and Materials Science, Faculty of Science, Chiang Mai University, Chiang Mai \\ 50200, Thailand \\ ${ }^{b}$ Department of Physics, Faculty of Science, Mahidol University, Bangkok 10400, Thailand \\ ${ }^{c}$ Department of Physics and Astronomy, University of Delaware, Newark, DE 19716, USA \\ ${ }^{d}$ Polar Research Institute of China, Pudong, Shanghai 200136, China \\ ${ }^{e}$ National Astronomical Research Institute of Thailand (NARIT), Chiang Mai 50180, Thailand \\ ${ }^{f}$ School of Natural Sciences, University of Tasmania, Hobart, Tasmania 7001, Australia \\ ${ }^{g}$ Department of Earth Science Education, Chonnam National University, Gwangju 61186, South Korea \\ E-mail: kledsai_p@cmu.ac.th, waraporn.n@cmu.ac.th, \\ david.ruf@mahidol.ac.th, evenson@udel.edu, jiangpeng@pric.org.cn, \\ pongpichit.ch@gmail.com, Marc.Duldig@utas.edu.au, \\ John.Humble@utas.edu.au, suyeonoh@jnu.ac.kr
}

The Galactic cosmic ray spectrum manifests subtle variations over the 22-year solar magnetic cycle in addition to more pronounced variations over the 11-year sunspot cycle. We conducted numerous latitude surveys by operating a neutron monitor onboard an icebreaker that traveled across a wide range of geomagnetic cutoff rigidity. Here we revisit our previous work to study spectral changes using 13 annual latitude surveys from 1994 to 2007 by comparing with neutron monitor data from Mawson instead of McMurdo, which closed in 2017, in order to allow a comparison with more recent latitude surveys. We confirm linear trends between count rates at different geomagnetic cutoff rigidity and changes in slope before and after the polarity reversal in 2000 as an effect of solar magnetic polarity. We performed two more latitude surveys (in 2019 and 2020) with a monitor similar to the 3NM64 in the previous surveys but without lead rings around the central tube, a so-called "semi-leaded neutron monitor." We also found similar results for the relationship between the count rate of the semi-leaded neutron monitor and that of the Jang Bogo and Mawson neutron monitor stations in Antarctica.

$37^{\text {th }}$ International Cosmic Ray Conference (ICRC 2021)

July 12 th - 23rd, 2021

Online - Berlin, Germany

\footnotetext{
${ }^{*}$ Presenter
} 


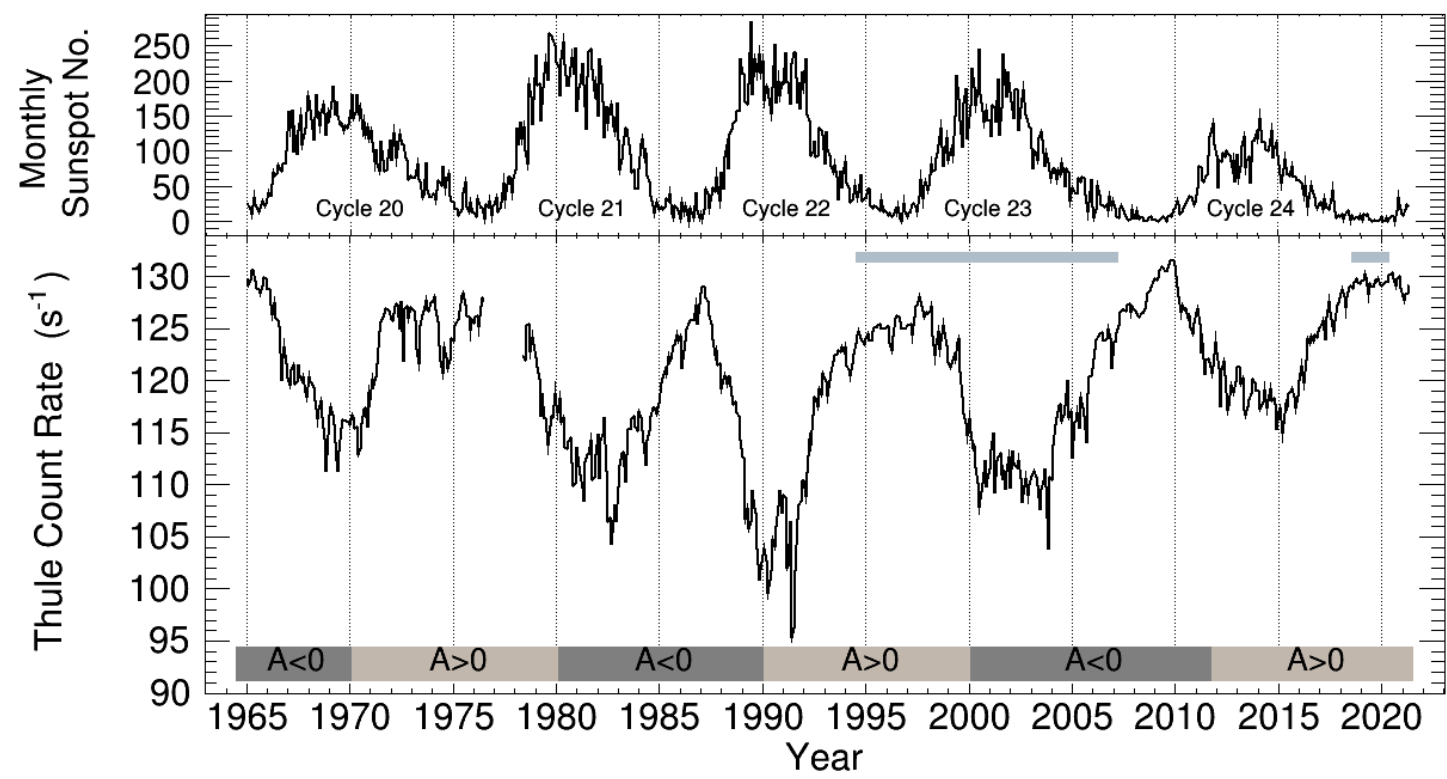

Figure 1: As solar activity rises (top panel, Source: WDC-SILSO Royal Observatory of Belgium, Brussels), the pressure-corrected count rate recorded by the neutron monitor in Thule decreases (bottom panel, Source: Bartol Research Institute, University of Delaware, USA). The solar magnetic polarity reversal can be seen between positive (denoted by $\mathrm{A}>0$ ) and negative (denoted by $\mathrm{A}<0$ ). This work presents observations for the periods 1994-2007 and 2019-2020, as indicated by horizontal bars between two panels.

\section{Introduction}

Galactic cosmic rays (GCRs) are high energy particles from outside the solar system arriving at the Earth. When GCRs enter the heliosphere, they encounter a turbulent magnetic field, causing significant variation in their intensity and energy. The influence of the sun on the intensity of GCRs is referred as Solar modulation. The GCR spectrum varies with the sunspot cycle and the solar magnetic cycle. The longest direct record of cosmic ray intensity comes from ground level observations from neutron monitors and muon detectors. Figure 1 shows solar activity in the top panel and a neutron monitor count rate in the bottom panel. It is clearly seen that the neutron monitor count rate is related to solar activity with 11-year cycle. This quasi-periodicity is convincingly reflected in the records of sunspots since the early 1600s and also in the galactic Cosmic ray intensity observed more directly since the 1950s.

The 11-year cycle of solar activity, characterized by the number of sunspots, is caused by evolution of the solar magnetic field. For example, during an active solar cycle, there can be several CMEs on a single day that sweep out high-energy particles from the Galaxy. That causes decreasing count rates because the neutron monitor typically responds to the $\mathrm{GeV}$ energy range of Galactic cosmic rays. The polarity of the large scale solar magnetic field also reverses approximately every 11 years. Unfortunately, we do not know exactly what cause these cycles, but the large scale reversal occurs when the magnetic field is most complex, near solar maximum. There are few sunspots during solar minimum but during solar maximum the number of sunspots increases, then drops down again to the next solar minimum. The counting rate recorded by a neutron monitor is inversely 
correlated with solar activity.

A mobile neutron monitor can record data rapidly through a wide range of geomagnetic cutoffs in a so-called latitude survey [1]. Such survey improve understanding of the geomagnetic cutoff rigidity (the minimum momentum per charge) is required for particles reaching a certain geographical location and track changes in the primary cosmic ray spectrum. Latitude surveys in 1976 and 1987 [2] and 1997 and 2006 [3] show an intersection of differential response function at rigidity $\approx 5-7 \mathrm{GV}$. The intersection of the two spectra is called the "crossover" measured at solar minima during epochs of opposite magnetic polarity. The crossovers can also be seen from the correlation of latitude survey neutron monitor count rates and count rates of fixed neutron monitors such as that at McMurdo station [3]. A regression analysis found a consistent trend with slopes that change when solar magnetic polarity flips, most likely due to a systematic change in the interplanetary diffusion coefficient for cosmic rays.

In recent years the neutron monitor at McMurdo has been moved to the Korean Jang Bogo station, and the mobile neutron monitor has been reconfigured. The series of 13 annual latitude surveys from 1994 to 2007 used by [3] therefore cannot be directly extended. In this work we repeat the analysis using neutron monitor data from the existing neutron monitor station, Mawson, instead of McMurdo station and confirm the crossovers. We also analyze two recent latitude surveys in 2019 and 2020 with a monitor similar to the 3NM64 in the previous surveys, but without the lead producer surrounded the central tube, the so-called "semi-leaded neutron monitor." We present this analysis using both the Mawson and Jang Bogo neutron monitor stations.

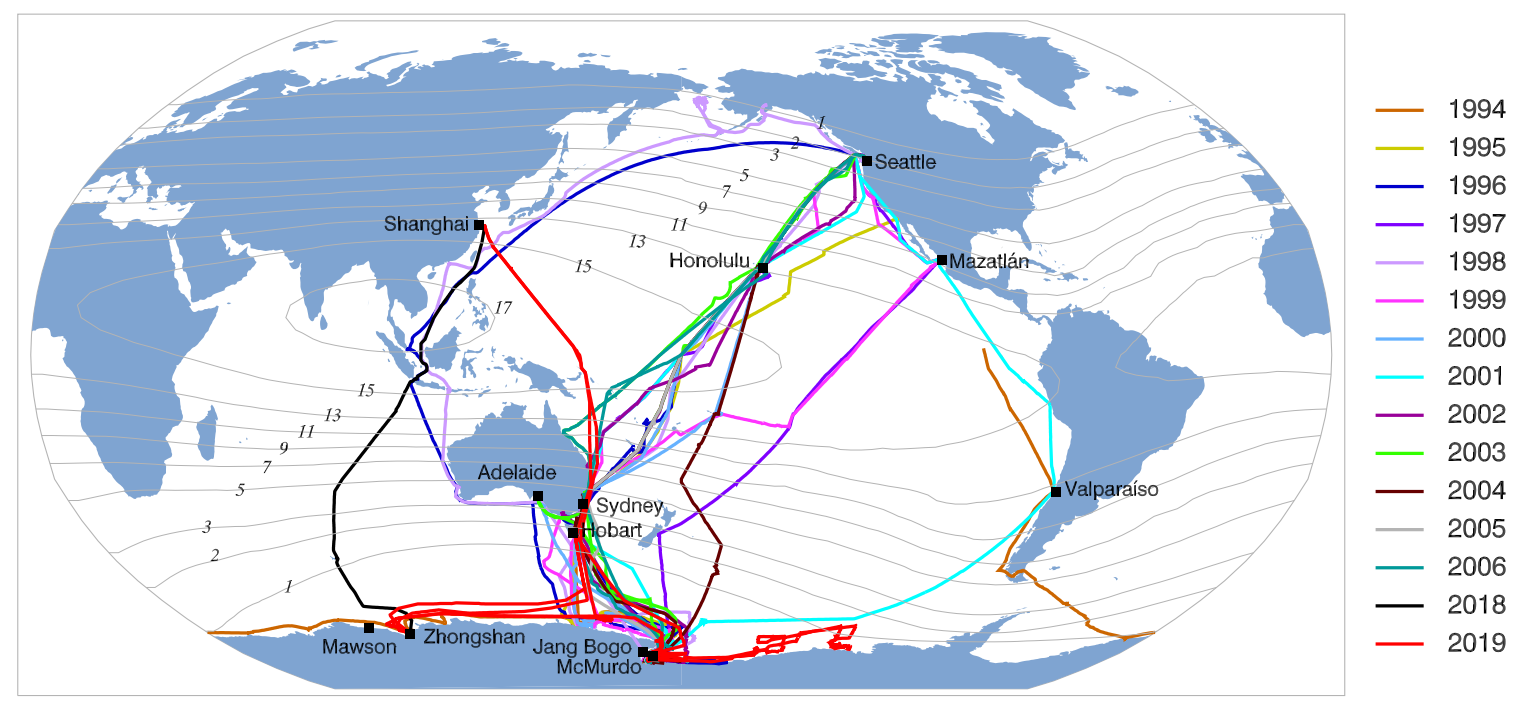

Figure 2: The track of the ship-borne neutron monitor latitude surveys for 1994-2007, and 2019-2020, superimposed on contours of the vertical cutoff rigidity (GV). [3] 


\section{Observations}

\subsection{Mobile neutron monitors}

The routes of mobile neutron monitors used in this work are shown in Figure 2. Each color indicates a "survey year," defined as the year in which the voyage began.

\subsubsection{Thirteen survey years, 1994-2007}

Surveys from 1994 to 2007 recorded data from three standard neutron monitors (3NM64) in a shipping container, nicknamed the "Tasvan." The mobile neutron monitor was operated aboard one of two U.S. Coast Guard icebreakers, the Polar Sea or the Polar Star, traversing the Pacific Ocean from Seattle, the USA, to McMurdo, Antarctica, and back on approximately six month voyages. In this analysis, we use count rate data from 13 survey years (1994-2007) corrected for pressure but uncorrected for short-term modulation variations with McMurdo count rate. We exclude times of large Forbush decreases $>10 \%$ documented in [3].

\subsubsection{Two survey years, 2018-2020}

Survey years 2018 and 2019 used two standard neutron monitors flanking one lead-free neutron monitor installed in a shipping container named "Changvan." The container was operated aboard the icebreaking vessel Xue Long, traveling from Shanghai to Zhongshan station in Antarctica. During the 2018 survey year (November 2, 2018 - March 11, 2019), the Changvan monitor collected data only on the return journey from Zhongshan station, Antarctica to Shanghai from 11 February -11 March 2019. In the 2019 survey year (October 21, 2019 - April 22, 2020), data were recorded both to and from Antarctica. For the whole survey data in 2018 and only the southbound data in 2019, we used count rate data provided in [4]. For additional data during the Northbound trip back to Shanghai in the 2019 survey year, we obtain it from Yakum et al. (in preparation).

\subsection{Fixed neutron monitor stations}

\subsubsection{Jang Bogo neutron monitor}

Jang Bogo is a Korean station located on the coast of Terra Nova Bay in Northern Victoria Land $\left(74.6^{\circ} \mathrm{S}, 164.23{ }^{\circ} \mathrm{E}\right)$, altitude $28 \mathrm{~m}$ from sea level, and vertical cutoff rigidity $<0.3 \mathrm{GV}$. The 18 -tube NM64 is installed near the cosmic ray lab. Jang Bogo station is $360 \mathrm{~km}$ away from McMurdo station, one of the United States stations. The pressure corrected count rate of the Jang Bogo neutron monitor used in this analysis came from the NMDB website with $\beta=0.9866 \% / \mathrm{mmHg}$ and the reference pressure $=733.56 \mathrm{mmHg}$ applied in the calculation. There was about 1 month (from January 5 to February 3) of data gap and several months of frequent missing data after February 19 from the NMDB website in 2020. This yields a small amount of data appearing in Figure 4(d).

\subsubsection{Mawson neutron monitor}

Mawson is located at the edge of the Eastern Antarctic plateau $\left(67.60{ }^{\circ} \mathrm{S}, 62.88^{\circ} \mathrm{E}\right)$. It is the first continental station and the longest continuously operating station south of the Antarctic Circle. The altitude is close to sea level, and vertical cutoff rigidity is 0.22 GV. From 1986 to October 16, 2002, a total of six neutron counters were placed in the same NM segment. After October 17, 
2002, the system was upgraded to use 18 counter tubes. Early in 2020, the data acquisition system was updated with new electronic firmware and computer software. We corrected Mawson data for pressure using barometric coefficient $\beta=0.9439 \% / \mathrm{mmHg}$ and the reference pressure 742.56 $\mathrm{mmHg}$. Analysis of Mawson data is complicated because of changes in the number of counter tubes in late 2002. Therefore, we explain some details for Mawson data processing in more detail.

\subsubsection{Data cleaning based on ratios of count rates}

From October 17, 2002, the number of counter tubes at Mawson increased from 6 to 18. The available data is the sum of the count rates for 6 tubes in the same section. We cleaned the data based on ratios of total count rate for each section. There are 3 sections, which refer to G0, G1, and G2. The count rates of the individual section were corrected by comparing section ratios with the averaged section ratios for the whole survey year. We refer to the survey averaged count rates as $\mathrm{A} 0$ for section $\mathrm{0}, \mathrm{A} 1$ for section 1, and $\mathrm{A} 2$ for section 2. If, for example, $\mathrm{G} 0$ rate is unavailable, the corrected count rate was calculated from $[(\mathrm{A} 0+\mathrm{A} 1+\mathrm{A} 2) /(\mathrm{A} 1+\mathrm{A} 2)] \mathrm{x}(\mathrm{G} 1+\mathrm{G} 2)$. If two sections were removed, the remaining section was used to calculate corrected data. If none of the tubes was operated, the count rate is presented as a data gap.

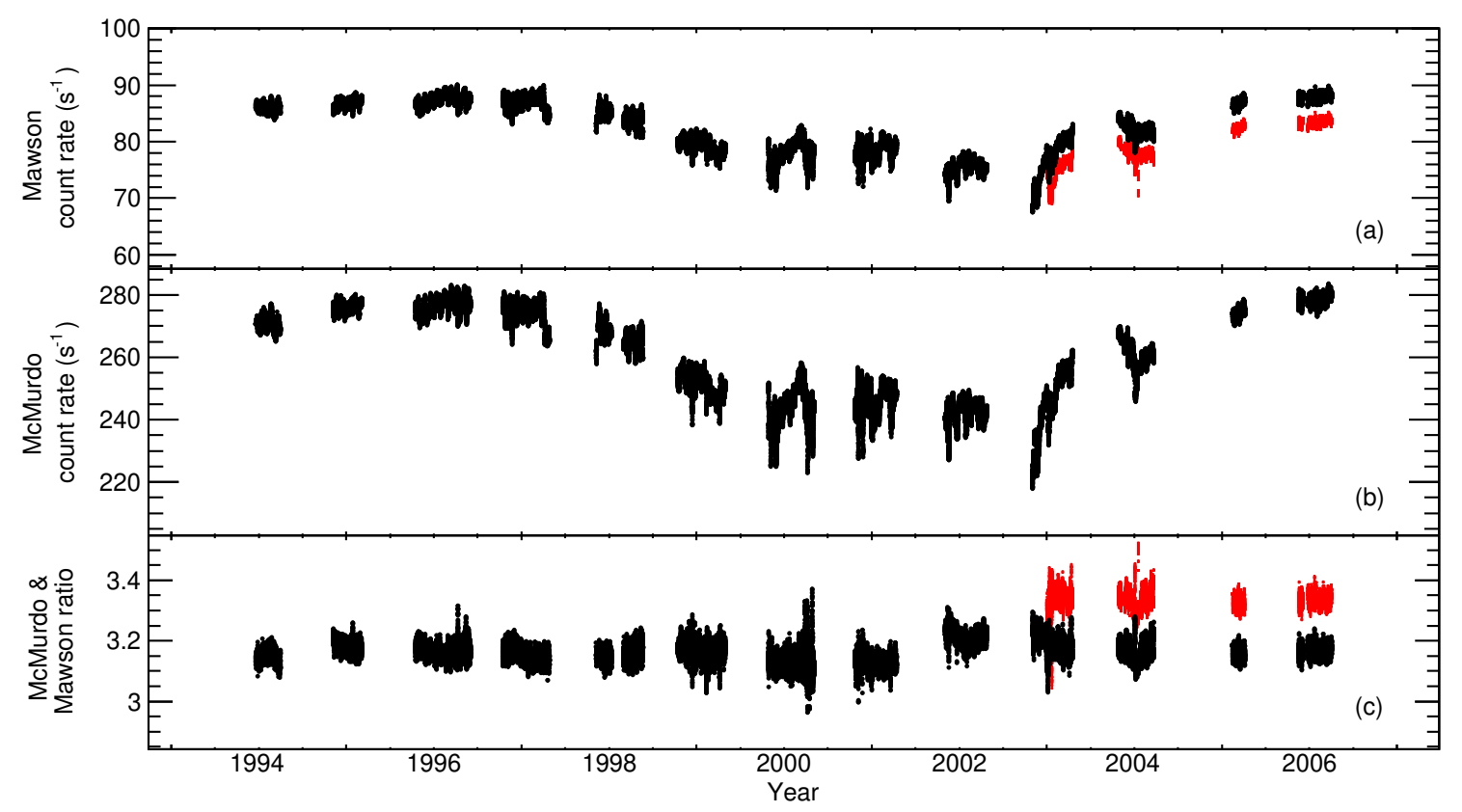

Figure 3: The count rate of 13 survey years from 1994 - 2007 at different fixed neutron monitor stations. (a) Mawson, (b) McMurdo, and (c) McMurdo/Mawson count rate ratio. Black dots indicate the data after normalization. Red dots represent the data before normalization.

\subsubsection{Mawson normalized with McMurdo}

A 6NM64 neutron monitor had initially operated Mawson station and later changed to 18NM64 from October 17, 2002. To study the change between the counting rates obtained from the mobile monitor and the fixed neutron monitor of different stations, we need to calibrate the detector. The method we use in this work is to use the ratio of the count rate between McMurdo and Mawson to 
find consistency. We calibrate Mawson count rates with McMurdo count rates because data from 13 survey years has been normalized with McMurdo [3]. Figure 3 (a)-(b) shows count rates of Mawson and McMurdo neutron monitor stations. Figure 3 (d) shows the count rate ratio of McMurdo Vs. Mawson. Here, we applied a normalization factor of 1.0533 to the uncorrected Mawson count rate (shown in red) from January 1, 2003, until the survey year 2006 ended. Black data points are the data that we use for analysis throughout this work.

\section{Consolidation of latitude survey data to study solar magnetic polarity effect}

Two results are described in this paper. First, we apply the regression analysis of [3] to the new survey data, comparing the mobile data both to Jang Bogo and Mawson. Second, we repeat the earlier analysis using Mawson in place of McMurdo.
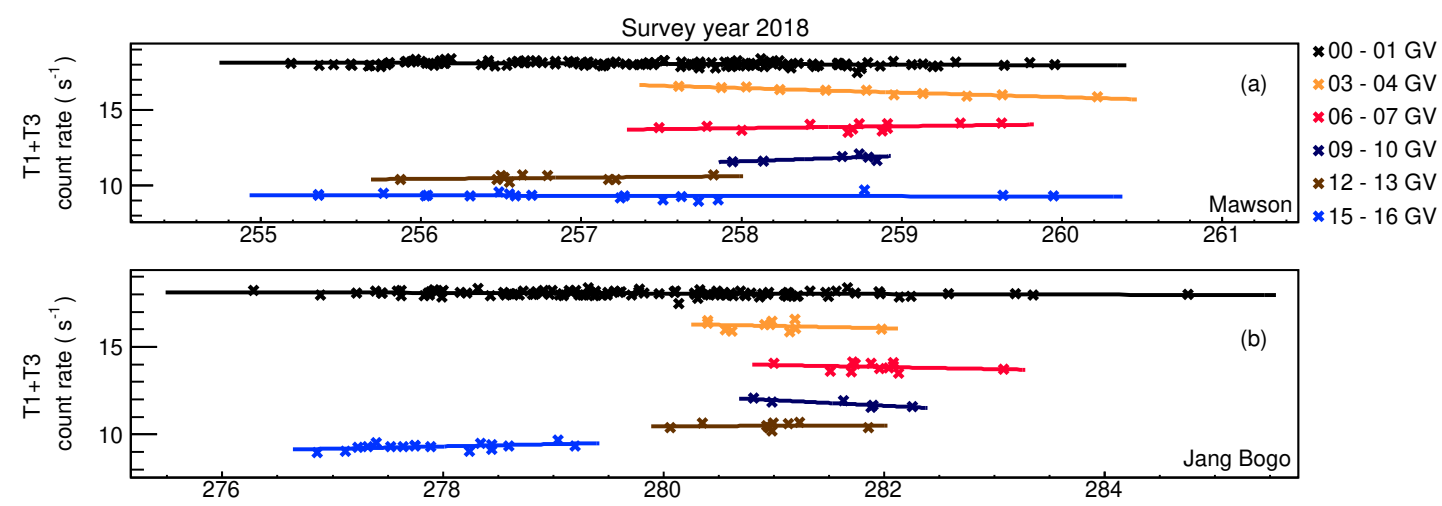

Survey year 2019
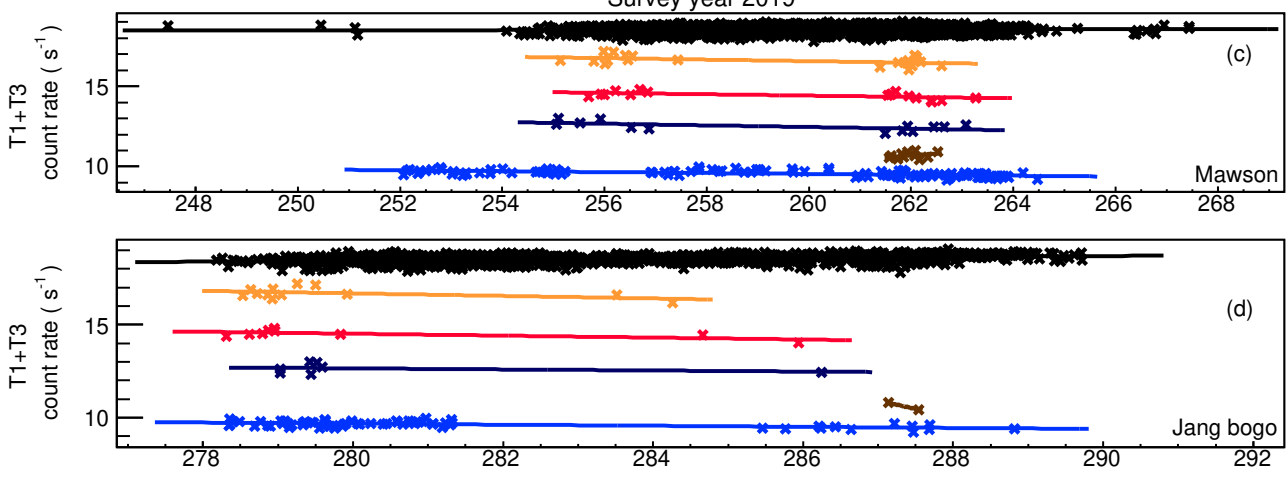

Figure 4: (a)-(b) Linear fits of mobile monitor count rate $\left(\mathrm{s}^{-1}\right)$ of sum $\mathrm{T} 1$ and $\mathrm{T} 3$ against Mawson and Jang Bogo hourly count rate $\left(\mathrm{s}^{-1}\right)$ for the 2018 survey year and (c)-(d) for the 2019 survey year for selected rigidity bins.

\subsection{Recent Surveys}

As there is no lead producer surrounding the central tube in these two surveys, we neglect data of this tube and use only the standard NM64s (T1\&T3) flanking the central tube for the analysis. We compare the ship-borne data to the two stationary stations Mawson and Jang Bogo, as the ship traversed close to those stations (see Figure 2). Figure 4 shows the regression of the $\mathrm{T} 1+\mathrm{T} 3$ count 
rates against the Mawson and Jang Bogo neutron monitor count rates for every third rigidity bin. The dots in Figure 4 indicate the hourly count rates of the neutron monitors. Our results demonstrate that the regression of semi-leaded neutron monitor count rates in survey years 2018-2019 vs. fixed station neutron monitor count rates can also be fitted by a straight line. Because Jang Bogo data are missing at various periods, for example, the big gap from January 2020 to June 2020, when it coincided with the ship-borne data, we then focus on the only analysis of Mawson data for the present surveys.

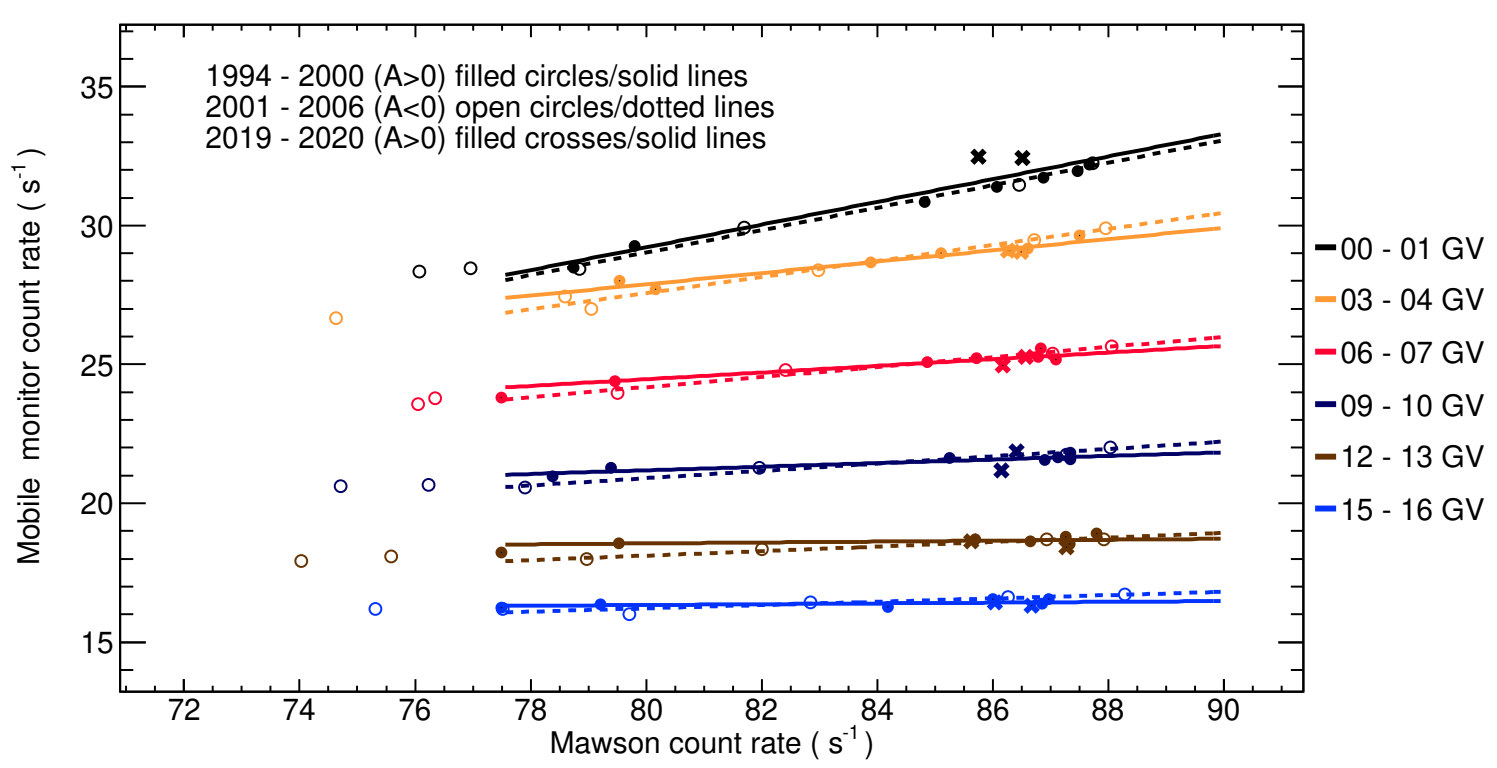

Figure 5: Regression of the mobile neutron monitor count rate in different apparent cutoff rigidity bins against the count rate of Mawson neutron monitor superimposing the data for different solar magnetic polarities. Every third bin has been selected for clarity corresponding to Figure 10 in [3] but comparing with Mawson instead of McMurdo data. Filled circles indicate average values over each survey year for positive $(A>0)$ solar magnetic polarity. Open circles present average values over each survey year for negative $(A<0)$ solar magnetic polarity, while the dotted lines are linear fits to these data. The survey year 2000 data is excluded from the regression analysis, as were all data with a Mawson count rate below $77.5 \mathrm{~s}^{-1}$; therefore, the transition period is excluded. Cross markers indicate data obtained from 2018 and 2019 survey years multiplied with the normalization factor described in Section 4. The solid lines showing the linear fits for positive $(A>0)$ solar magnetic polarity of fill circles and cross markers for each rigidity bin in regression analysis.

\subsection{Earlier Surveys}

We used 1994-2007 data from [3] corrected for pressure and uncorrected for short-term variation and also removing Forbush decrease events. Mobile monitor count rate data were divided into $1 \mathrm{GV}$ width bins in apparent cutoff rigidity (i.e., $0-1 \mathrm{GV}, 1-2 \mathrm{GV}, \ldots, 16-17 \mathrm{GV}$, and over $17 \mathrm{GV}$ ) and plotted against the count rate of Mawson neutron monitor, as shown (for every third rigidity bin) in Figure 5 including all the survey years for both solar magnetic polarity states. Figure 5 should be compared with Figure 10 in [3]. The regression for each rigidity bin against Mawson can be fitted 
by a straight line. We confirm the change in the slope before and after solar polarity reversal in the year 2000 .

\section{Results and Discussions}

To compare the two tubes in the recent survey years to the 3NM64 in a 13-year survey, we apply a normalization factor of 1.80 for the survey year 2018 and that of 1.75 for the survey year 2019 to T1+T3. We obtain two data points for each rigidity bin which have been added to Figure 5 . Our results confirm linear trends between count rates at different geomagnetic cutoff rigidity and changes in slope before and after the polarity reversal in 2000 as an effect of solar magnetic polarity similar to the results shown in [3]. Results from two recent latitude surveys are consistent with the previous conclusions.

\section{Acknowledgments}

We acknowledge logistical support from Australia's Antarctic Program for operating the Mawson NM. The research is supported in part by Thailand Science Research and Innovation via Research Team Promotion Grant RTA6280002. We thank the Northern Science Park (Chiang Mai) for providing laboratory space at the park, which helps the research team working smoothly.

\section{References}

[1] J. Bieber, J. Clem, M. Duldig et al., Latitude survey observations of neutron monitor multiplicity, J. Geophys. Res 109 (2004) .

[2] H. Moraal, M.S. Potgieter, P.H. Stoker et al., Neutron monitor latitude survey of cosmic ray intensity during the 1986/1987 solar minimum, J. Geophys. Res. Space Phys 94 (1989) 1459.

[3] W. Nuntiyakul, P. Evenson, D. Ruffolo et al., Latitude Survey Investigation of Galactic Cosmic Ray Solar Modulation during 1994-2007, Astrophys J 795 (2014) 11.

[4] S. Khamphakdee, P. Jiang, P. Chuanraksasat et al., Preliminary analysis of the changvan neutron monitor operation in latitude surveys during 2019-2020, J. Phys.: Conf. Ser 1719 (2021) 012010. 